\title{
Zur Verkehrsbezeichnung von Döner Kebap und dönerähnlichen Produkten bei Abgabe in Fertigpackungen und in loser Form
}

\author{
Dr. C. Dildei' und Dr. H. Kirchhoff ${ }^{2}$ \\ ${ }^{1}$ Niedersächsisches Landesamt für Verbraucherschutz und Lebensmittelsicherheit (LAVES), Veterinärinstitut Hannover, \\ Eintrachtweg 17, 30173 Hannover \\ ${ }^{2}$ Niedersächsisches Landesamt für Verbraucherschutz und Lebensmittelsicherheit (LAVES), Lebensmittelinstitut Oldenburg, \\ Martin-Niemöller-Str. 2, 26133 Oldenburg
}

Es wird ein dreistufiges Bezeichnungsschema zur Diskussion und Abstimmung gestellt, das sich am Grad der Leitsatzkonformität der zu beurteilenden Erzeugnisse orientiert. Von „vollständig leitsatzkonformen“ Döner-Kebap-Spießen werden solche mit „überwiegend leitsatzkonformen“ und solche mit „geringen bzw. fehlenden leitsatzkonformen“ Produkteigenschaften unterschieden und entsprechend bezeichnet.

1. Döner Kebap: ausschließlich leitsatzkonforme Produkteigenschaften.

2. Hackfleisch-Drehspieß (o. Ä.) z. B. nach Döner Kebap-Art: überwiegend leitsatzkonforme Produkteigenschaften.

3. Hackfleisch-Drehspieß (o. Ä.): Keine oder nur geringe leitsatzkonforme Produkteigenschaften. Hier handelt es sich um ein ALIUD (Erzeugnis eigener Art).

Der Begriff „Grad der Leitsatzkonformität“ im Sinne von „überwiegend leitsatzkonforme Produkteigenschaften“ bezieht sich auf die qualitative und quantitative Zusammensetzung des Fleischanteils. Er wird verwendet zur Charakterisierung von dönerähnlichen Erzeugnissen im Sinne von „... nach Döner Kebap-Art".

Enthält der Hackfleisch-Anteil eines ansonsten in der Zusammensetzung leitsatzkonformen „Döner Kebap“-Spießes Bindemittel wie z. B. Stärke und/oder Paniermehl, bestehen nur noch überwiegend leitsatzkonforme Produkteigenschaften. Die Beschreibung könnte „Drehspieß nach Döner Kebap-Art mit Bindemitteln“ lauten. Die Angabe „Döner Kebap mit Bindemitteln“ ist nicht zulässig.

Nur noch überwiegend leitsatzkonforme Produkteigenschaften bestehen auch bei Anteilen von mehr als 60\% Hackfleisch. Für solche Erzeugnisse sollten Beschreibungen wie z. B.
„Hackfleisch-(Dreh-)spieß nach Döner Kebap-Art“, „Drehspieß aus Hackfleisch nach Döner Kebap-Art“ oder „Drehspieß mit x\% Hackfleisch nach Döner Kebap-Art“ verwendet werden. Ausreichend kenntlich gemacht ist ein überhöhter Hackfleischanteil auch durch die alleinige Bezeichnung „HackfleischDrehspieß“. Die Angabe „Döner Kebap mit 80\% Hackfleisch“ ist nicht zulässig.

Werden zudem Bindemittel verarbeitet, handelt es sich um ein „Erzeugnis eigener Art“ (ALIUD) im Sinne einer „Hackfleischzubereitung am Spieß“, da nur noch geringe leitsatzkonforme Produkteigenschaften bestehen.

\section{Zusammenfassung/Erläuterung}

Aufgrund der Problematik, dass Drehspiess-Erzeugnisse unterschiedlicher Zusammensetzung insbesondere bei Abgabe als lose Ware als „Döner“ bezeichnet werden, obwohl sie z. B. aufgrund des hohen Hackfleischanteils und der Verwendung von Bindemitteln nicht leitsatzkonform und somit nicht dem klassischen Döner Kebap entsprechen, wird eine bundesweit abgestimmte Beurteilungspraxis im Sinne eines dreistufigen Bezeichnungsschemas vorgeschlagen.

\section{Diskussionsergebnis}

Frau Dildei und Herr Kirchhoff sammeln die Voten der Bundesländer zur oben genannten Problematik der Beurteilung von „Döner Kebab“, fassen das Ergebnis dieser Diskussion unter Festlegung einer angemessenen Frist im FIS-VL unter „Newsgroup“ zusammen und stellen es zur Abstimmung. 